\title{
Transmitting Data inside a Hole: Recent Advances in Hollow Core Photonic Bandgap Technology
}

\author{
F. Poletti*, J.P. Wooler*, N.V. Wheeler*, N.K. Baddela*, D.R. Gray*, E. Numkam Fokoua*, J.R. Hayes*, \\ Y. Jung*, S.U. Alam*, S.R. Sandoghchi*, V.A.J.M. Sleiffer**, M. Kuschnerov***, M.N. Petrovich*, and \\ D.J. Richardson* \\ *Optoelectronics Research Centre, University of Southampton, Highfield, Southampton, SO17 1BJ, UK \\ ** COBRA institute, Eindhoven University of technology, Eindhoven, The Netherlands \\ *** Coriant GmbH, St-Martin-Str. 76, Munich, Germany \\ frap@orc.soton.ac.uk
}

\begin{abstract}
We review our recent progress in the fabrication, characterization, modeling and splicing of wide transmission bandwidth hollow core photonic bandgap fibers and discuss their modal properties and potential for data transmission.

OCIS codes: (060.4005) Microstructured fiber; (060.0060) Fiber optics and optical communications.
\end{abstract}

\section{Introduction}

Hollow core photonic bandgap fibers (PBGFs) represent one of the most radical approaches to address fundamental limits of loss and nonlinearity in transmission fibers [1]. Wave-guidance in air with less than $0.1 \%$ of optical power overlap with the glass can be achieved by surrounding a large hollow core (15-25 times larger than the operating wavelength) with a periodic photonic crystal cladding. The small overlap with the glass structure not only offers orders-of-magnitude reduction in the optical nonlinearity, but also allows light propagation at speeds close to the vacuum speed of light, which has appealing advantages for a growing number of ultra-low latency applications [2]. To be able to exploit the unique characteristics of PBGFs, significant further improvements need to be achieved, not only in the loss of these fibers but also in their modal properties. Here we discuss our progress in developing PBGFs suitable for data transmission.

\section{Wide Bandwidth Operation through Surface Mode Elimination}

While PBGFs with $<2 \mathrm{~dB} / \mathrm{km}$ loss were demonstrated as far back as in 2004 [3], the early transmission experiments on PBGFs indicated strong modal and polarization instabilities which we would now retrospectively attribute to the unsuppressed guidance of surface modes (SMs) at wavelengths close to those used for data transmission. SMs, are physically located at an incorrectly terminated or distorted core surround and are very susceptible to structural and environmental perturbations. Their interaction with air-guided core modes can cause significant reduction in the useable transmission bandwidth and severe limitations in the achievable data transmission performance [4]. For this reason we have focused our recent fabrication efforts on the management of SMs and on the development of recipes to produce fibers with a thin and carefully controlled core surround [5]. Although potentially more susceptible to deformations and drawing instabilities, especially in fibers with large core sizes, once perfected, such an approach can lead to the realization of fibers with a combination of low-loss, wide bandwidth and modal qualities that are adequate for data transmission. Recently we have reported two PBGFs with such characteristics: a 19 cell core fiber with a SM-free bandwidth in excess of $150 \mathrm{~nm}$ and loss of $3.5 \mathrm{~dB} / \mathrm{km}$ [2], and a 37 cell core fiber with a loss of 3.3 $\mathrm{dB} / \mathrm{km}$ and strong robustness to bending despite its very large mode field diameter of $\sim 23 \mu \mathrm{m}[6]$, see Fig.1.
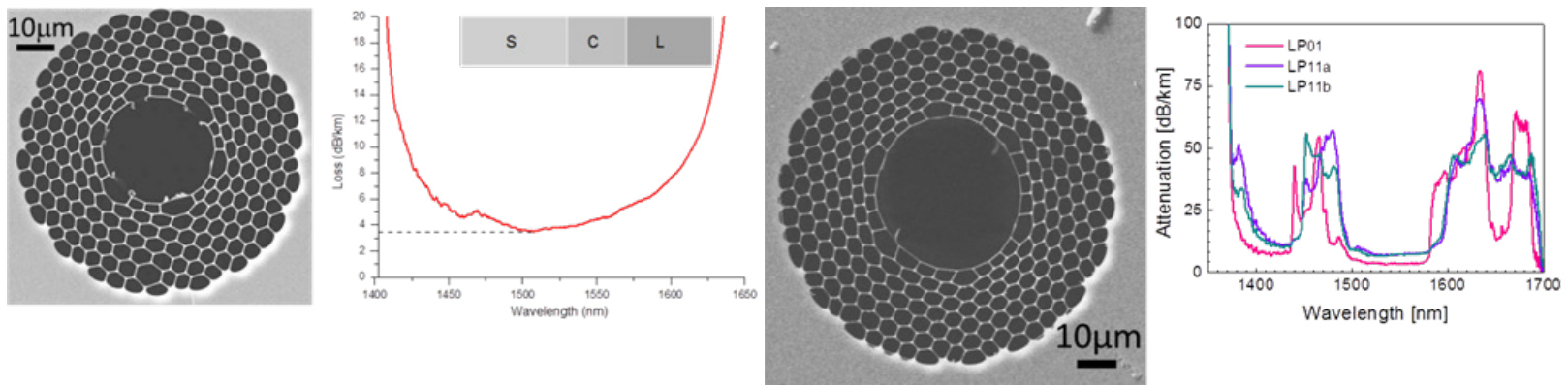

Fig.1. SEM and cutback loss of: (left) a 19 cell PBGF with a minimum fundamental mode loss of $3.5 \mathrm{~dB} / \mathrm{km}$ and a $3 \mathrm{~dB}$ bandwidth of $160 \mathrm{~nm}$ [4]; (right) a 37 cell PBGF (shown in the same scale) with a loss of 3.3 and $\sim 7 \mathrm{~dB} / \mathrm{km}$ for the $\mathrm{LP}_{01}$ and $\mathrm{LP}_{11}$ modes, respectively [6]. 


\section{Data Transmission Experiments}

Large core PBGFs can guide several vector modes. However, due to a very low intermodal cross-talk, through selective excitation of the fundamental mode only and spatial filtering at the receiver-end, they can also operate as effectively single mode fibers. In this way we have succeeded in transmitting $37 \times 40 \mathrm{Gbit} / \mathrm{s}$ DWDM on-off keyed channels in the C-band over 250m of the 19 cell fiber shown in Fig.1 [2]. The very low power penalties observed in this experiment indicated the possibility to further increase the overall transmitted capacity by exploiting both polarizations, increasing the number of DWDM channels and using more spectrally efficient coherent modulation formats. In this way, using 32QAM $(2 \times 96 \times 160 \mathrm{Gbit} / \mathrm{s} / \mathrm{channel} / \mathrm{pol}$. $)$ we have subsequently transmitted down the same fiber up to $30.7 \mathrm{Tbit} / \mathrm{s}$ [7]. A further step to enhance the transmitted data capacity is that of using MIMO-aided space division multiplexing (SDM) schemes, where one excites and transmits cleanly and independently several modes through the PBGF. Using the 3 lower spatial modes (in both polarizations) and 96 DWDM channels modulated with a 16 QAM format, we have recently demonstrated a total data rate of $73.7 \mathrm{~Tb} / \mathrm{s}$ over $310 \mathrm{~m}$ of the 37 cell PBGF shown in Fig.1. This currently stands as the highest data rate ever transmitted through a PBGF [6].

\section{PBGF Self-Splicing}

A mandatory requirement for PBGFs to be practically useable as transmission fibers is the possibility to achieve robust and low loss splices both with themselves and with solid fibers. Although in principle this is a challenging problem, as it requires applying sufficient heat to fuse the solid cladding together without causing collapse in the thin microstructured cladding, we have recently demonstrated a splicing recipe to achieve it. Using a sequence of tack, sweep and short arc pulses with a standard fusion splicer we have achieved robust self-splices with a minimum and average loss of 0.07 and $0.16 \mathrm{~dB}$, respectively (Fig.2), and a negligible impact on the fiber's high order mode content [8]. Subsequent data transmission tests over $>1 \mathrm{~km}$ of PBGFs containing splices fabricated with this recipe have shown no significant additional power penalty [9]. The same recipe, recently applied to solid-PBGF splices has produced robust splices with only minor microstructure deformation and hence reduced loss (Fig.2c,d).
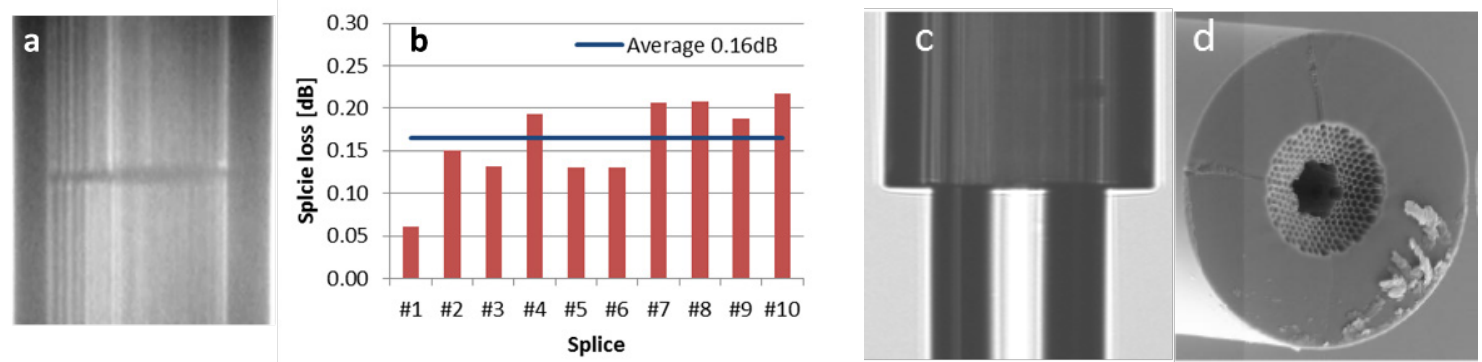

Fig.2. (a) Two PBGFs spliced with the multi-step recipe of Ref. [9] showing no distortion in the microstructure and a $\sim 5 \mu \mathrm{m}$ residual air gap; (b) Splice loss for 10 different realizations (min: $0.07 \mathrm{~dB}$, average $0.16 \mathrm{~dB}$ ); (c) solid to PBGF splice; (d) broken splice showing a good microstructure integrity.

\section{Conclusions}

We have presented some of our latest results in the development of hollow core PBGFs suitable for high capacity data transmission. We have developed recipes to produce fibers with an uninterrupted transmission bandwidth covering the $\mathrm{S}+\mathrm{C}+\mathrm{L}$ bands and with realistic prospects of significant further expansion. Once surface modes are eliminated, the air-guided modes of these PBGFs are "well behaved" and their intermodal cross-talk is low enough to allow high capacity single mode transmission. MIMO-assisted SDM can also be achieved to boost-up the total capacity. Finally, we have shown that these fibers can be robustly spliced with low-loss using conventional arcfusion splicers. Although much work remains to be done, we believe that the very encouraging progress achieved over the last two years indicates a bright future for PBGF technology.

\section{Acknowledgment}

This work was supported by the EU 7th Framework Programme under grant agreement 258033 (MODE-GAP) and by the UK EPSRC through grant EP/H02607X/1.

\section{References}

[1] R. F. Cregan et al., Science,285, 1537, (1999)

[2] F. Poletti et al., Nature Photonics, 7, 279-284(2013)

[3] B. J. Mangan et al., Proc OFC'04, PDP24

[4] F. Poletti et al., Opt. Express 13, 9115 (2005)

[5] R.Amezcua-Correa et al., Opt.Express 15, 17577 (2007)
[6] Y. Jung et al., Proc OFC'13, PDP5A.3

[7] V. A. J. M. Sleiffer et al., Proc OFC'13, OW1I.5

[8] J. Wooler et al., Proc OFC'13, OM3I.

[9] J. Wooler et al., ECOC'13, (submitted) 